\title{
Referenciação dos Cuidados de Saúde Primários ao Serviço de Urgência de Pediatria de um Hospital Nível II: Que Realidade?
}

\section{Reference of Primary Health Care to the Pediatric Emergency Department at a Level II Hospital: What Reality?}

Filipa Vale ${ }^{1 *}$, Filipa Pinto ${ }^{2}$, Rui Oliveira ${ }^{3}$, Cecília Martins $^{4}$, Susana Lopes ${ }^{5}$

\section{RESUMO}

INTRODUÇÃO: O objetivo foi caracterizar os episódios referenciados pelos cuidados de saúde primários (CSP) ao serviço de urgência (SU) do Centro Hospitalar do Médio Ave, assim como classificar a adequabilidade da referenciação.

MATERIAL E MÉTODOS: Foi realizado um estudo observacional retrospetivo, incluindo todos os utentes com idade inferior a 18 anos referenciados por parte dos CSP ao SU, durante o ano de 2017. A referenciação foi considerada adequada sempre que foram realizados exames complementares de diagnóstico ou terapêuticas não disponíveis nos CSP. Assim como, a observação por outra especialidade que não a pediatria, a necessidade de internamento e a transferência para outro hospital.

RESULTADOS: Foram obtidos 406 episódios de urgência, dos quais 25,9\% obtiveram como diagnóstico de saída a patologia gastrointestinal e 29,4\% a patologia respiratória. Foram consideradas adequadas $65,3 \%$ das referenciações, sendo as mais frequentes a patologia gastrointestinal, respiratória e trauma. As patologias genitourinária e infecciológica foram as mais constantes quanto à referenciação inadequada ( $O R=11,5$ e $O R=3,49$, respetivamente, ajustado à idade). Verificou-se concordância entre o motivo de referenciação e o diagnóstico de saída nas patologias gastrointestinal, otorrinolaringológica, trauma e oftalmológica (kappa > 0,7). A grande maioria (92,2\%) das crianças teve alta clínica.

1. Interna de Medicina Geral e Familiar, Unidade de Saúde Familiar Antonina, Vila Nova de Famalicão, Portugal. 2. Interna de Pediatria, Centro Hospitalar Médio Ave, Santo Tirso, Portugal. 3. Assistente de Medicina Geral e Familiar, Unidade de Saúde Familiar Antonina, Vila Nova de Famalicão, Portugal. 4. Assistente Hospitalar Graduada Pediatria, Centro Hospitalar Médio Ave, Santo Tirso, Portugal. 5. Assistente Hospitalar Pediatria, Centro Hospitalar Médio Ave, Santo Tirso, Portugal. Recebido/Received: 22/02/2021 - Aceite/Accepted: 30/08/2021 - Publicado online/Published online: 17/09/2021 - Publicado/Published: 30/09/2021

- Autor (es) (ou seu (s) empregador (es)) e Gazeta Médica 2021. Reutilização permitida de acordo com CC BY-NC. Nenhuma reutilização comercial. ${ }^{\circ}$ Author(s) (or their employer(s)) and Gazeta Médica 2021. Re-use permitted under CC BY-NC. No commercial re-use. 
CONCLUSÃO: A taxa de referenciação adequada aos serviços hospitalares no presente estudo foi semelhante à descrita na literatura. No entanto, a otimização da comunicação entre os CSP e o SU, a implementação de protocolos, bem como, a formação dos profissionais dos CSP permitirão melhorar a referenciação.

PALAVRAS-CHAVE: Criança; Cuidados de Saúde Primários; Encaminhamento e Consulta; Serviço de Urgência Hospitalar

\section{ABSTRACT}

INTRODUCTION: Our objective was to characterize the situations referred to the emergency department by the primary health care as well as assess the pertinence of this referral.

METHODS: A study realized was observational, retrospective and included patients were younger than 18 years old referred to the emergency department by primary health care, over 2017. The referral was considered appropriate when complementary exams or therapeutics were performed, which were not available at the primary health care, as well as observation by other medical speciality but paediatrics, admission necessity or transfer to other hospital were needed.

RESULTS: A total of 406 emergency episodes were obtained. The most frequent diagnoses were respiratory disease (29.4\%) and gastrointestinal disease (25.9\%). A percentage of 65.3\% of referrals were considered adequate and the most common pathologies were gastrointestinal and respiratory disease and trauma. The genitourinary $(\mathrm{OR}=11.5)$ and infectious $(O R=3.49)$ pathologies were consistent on inadequate referral considering patients' age. A match between referral's reason and final diagnosis regarding gastrointestinal pathologies, otolaryngology pathologies and ophthalmic pathologies, as well as trauma (kappa > 0.7), was observed. The majority (92.2\%) of the cases was discharged from the hospital.

CONCLUSION: The hospital's adequate referral rate was similar to the one described in other studies. However, communication's optimization between the primary health care units and the emergency department, as well as more professional training, are vital to improve this referral.

KEYWORDS: Child; Emergency Service, Hospital; Primary Health Care; Referral and Consultation

\section{INTRODUÇÃO}

O Centro Hospitalar do Médio Ave (CHMA) é constituído por duas unidades físicas, a unidade de Vila Nova de Famalicão (UF) e a unidade de Santo Tirso (UST), e tem como área de influência os concelhos de Vila Nova de Famalicão, Santo Tirso e Trofa. Estes concelhos têm uma população residente estimada de cerca de 238690 habitantes, dos quais 44210 têm idade igual ou inferior a 19 anos, segundo os dados do Instituto Nacional de Estatística (INE) referentes ao ano de 2017. ${ }^{1}$ O serviço de urgência do CHMA, em Famalicão, oferece apoio a todos os utentes em idade pediátrica dos três concelhos acima referidos.

Nesta região existe um total de dezanove Unidades de Saúde Familiar (USF), oito unidades de cuidados de saúde personalizados (UCSP) e dois serviços de atendimento permanente (SAP), do ACeS Ave/Famalicão e do ACeS Grande Porto I - Santo Tirso/Trofa.

Os cuidados de saúde primários (CSP), através das con- sultas de saúde infantil e consulta aberta, garantem não só o seguimento regular dos utentes pediátricos, mas também a resolução e orientação de situações agudas. ${ }^{2}$ A maioria dos CSP oferecem ao utente assistência de 12 horas por dia (das 08:00 às 20:00 horas) nos dias úteis, e os SAP prestam apoio aos fins-de-semana e feriados (das 08:00 às 14:00 horas). Relativamente a recursos disponíveis, estes são similares entre as várias unidades de CSP, no que respeita a exames complementares de diagnóstico (ECD) e terapêuticas. Nomeadamente a realização de teste diagnóstico antigénio rápido (TDAR) para Streptococcus do grupo A, tira-teste urinária ou exames microbiológicos, assim como a possibilidade de medicar com penicilina intramuscular (IM), corticoide, ondansetrom, anti-histamínico ou analgésico/antipirético oral.

Os lactentes, as crianças e adolescentes com patologia aguda são assistidos nos CSP em situação aguda e, quando entendido pelo clínico, são referenciadas para o serviço de urgência (SU) hospitalar, com o objetivo de 
realizar exames complementares e/ou terapêuticas não disponíveis nos CSP, ou ainda para avaliação e orientação pela especialidade de pediatria ou de outras especialidades.

A procura dos serviços de urgência pediátricos tem aumentado nos últimos anos, devendo-se principalmente à iniciativa própria do utente e/ou dos seus familiares. ${ }^{3-5}$ Os principais motivos para a utilização da urgência pediátrica, por iniciativa dos familiares/cuidadores, prendem-se com a perceção de gravidade da doença, a noção de maior especialização do pediatra e da maior facilidade no acesso à realização de exames diagnóstico. ${ }^{6-8}$

Além desta procura direta pelos serviços de urgências, existe também, descrita na literatura, uma referenciação inadequada para estes serviços por parte dos CSP. 2,4,5 Os autores consideram importante compreender a realidade da situação na sua comunidade, bem como propor estratégias para minorar estes números e desta forma promover uma melhor articulação entre os CSP e os cuidados hospitalares. Será urgente a formação aos CSP? Em que áreas?

Este estudo pretende analisar os principais motivos de referenciação por parte dos CSP, na comunidade em estudo, bem como caracterizar os episódios ao SU do CHMA e verificar a adequabilidade da referenciação.

\section{MATERIAL E MÉTODOS}

Foi realizado um estudo observacional, retrospetivo, incluindo todos os utentes com idade inferior a 18 anos que recorreram ao SU de pediatria no CHMA, referenciados pelos CSP, durante o ano de 2017.

As variáveis analisadas foram: o número de episódio, a idade, o género, o local de proveniência (USF/UCSP do ACeS Ave/Famalicão e Santo Tirso/Trofa, SAP Famalicão e Santo Tirso e fora destes ACeS), o motivo de referenciação, a triagem de Manchester, os exames complementares de diagnóstico (ECD), as terapêuticas efetuadas, assim como a observação por outra especialidade hospitalar, o diagnóstico principal na alta hospitalar e a orientação na alta (internamento, outro hospital e alta médica). $\bigcirc$ motivo da consulta foi definido com base na triagem de Manchester e na consulta do episódio.

Tanto o motivo da consulta como o diagnóstico principal foram analisados de acordo com a codificação International Classification of Diseases 10th Revision (ICD-10), a mesma utilizada pelo CHMA. Sempre que no motivo de referenciação fosse descrita mais que uma queixa, dentro do mesmo grupo de patologia, estas foram agrupadas; por exemplo no caso da menção de náuseas, vómi- tos e dor abdominal no mesmo episódio, estes sintomas foram agrupados em sintomas gastrointestinais (GI), e no caso de febre, tosse e dispneia foram agrupados em sintomas respiratórios.

Os autores consideraram como referenciação adequada quando um dos seguintes critérios se aplicava: requisição de ECD não disponíveis nos CSP (exame de imagem, estudo analítico e eletrocardiograma (ECG)); instituição de terapêutica exceto a disponível nos CSP (penicilina intramuscular (IM), corticoide oral, analgésico ou antipirético bem como o anti-histamínico oral). Assim como casos em que a terapêutica estaria disponível, mas exigia uma reavaliação a curto prazo, não sendo exequível essa abordagem no âmbito de consulta aberta das unidades avaliadas, como por exemplo terapêutica broncodilatadora, a terapêutica oral com ondansetrom, o enema, a terapêutica endovenosa (EV), são atitudes terapêuticas que exigem a vigilância ativa, e esta não pode ser realizada no domicílio. Sempre que foi realizada a observação por outra especialidade e a necessidade de internamento ou transferência para outro hospital foi também considerada como referenciação adequada.

No que concerne à referenciação inadequada, os autores definiram todas as situações em que o ECD e a terapêutica estavam disponíveis nos CSP e que não exigiam reavaliação urgente após o momento da consulta como o teste diagnóstico antigénico rápido (TDAR) para Streptococcus do grupo A, tira-teste urinária, exames microbiológicos, a penicilina intramuscular (IM), terapêutica com corticoide oral, paracetamol, bem como outra medicação oral.

A recolha de dados foi realizada no Microsoft ${ }^{\circledR}$ Excel 2019, tendo em consideração o número de episódio, e a análise estatística foi efetuada no SPSS ${ }^{\circledR}$ versão 26. As variáveis contínuas foram caracterizadas pela média ( \pm desvio padrão) ou mediana (mínimo-máximo) de acordo com a distribuição simétrica ou assimétrica, respetivamente; e as variáveis categóricas por frequências absolutas e relativas. Para comparar variáveis contínuas foi usado o teste não paramétrico Mann Whitney. O teste de Qui-quadrado e o teste exato de Fisher foram usados para comparar variáveis categóricas, o último quando o valor observado foi inferior a 5. Foi efetuada uma análise multivariada por regressão logística ajustada à idade para avaliar a adequabilidade da referenciação. O coeficiente de concordância de kappa foi utilizado para avaliar a concordância entre o motivo de referenciação e o diagnóstico de saída. Assumiu-se como valor de kappa $<0$, uma concordância insignificante, como valor entre 0 - 0,2, uma concordância fraca, entre 0,21 - 0,4 razoável, entre 0,41 - 0,6 moderada, entre 0,61 - 0,8 uma concor- 
dância forte e entre 0,81 - 1 quase perfeita. Um valor de p inferior a 0,05 foi considerado estatisticamente significativo. Este estudo foi aprovado pela comissão de ética e conselho de administração do CHMA.

\section{RESULTADOS}

No ano de 2017 ocorreram um total de 406 episódios de urgência referenciados a partir dos CSP, correspondendo a 1,3\% do total de episódios de urgência nesse ano (31 399). Excluíram-se oito episódios da análise por abandono do utente do SU. A mediana de idades da amostra foi de 6 anos, predominando o grupo entre os 1 e os 2 anos de idade (14,6\%).

As características da amostra comparando entre a referenciação adequada e inadequada por parte dos CSP encontra-se descrita na Tabela 1.

A maioria (82,7\%) dos utentes foram referenciados por USF/UCSP, 2,8\% através do SAP e os restantes (2,8\%) provenientes de fora do ACeS. De acordo com a triagem de Manchester, 5,3\% foram considerados como muito urgentes, 65,1\% como urgentes e 28,9\% como pouco urgentes.

Os motivos de referenciação mais frequentes foram as queixas do foro gastrointestinal (GI) (25,9\%), a patologia respiratória (19,3\%), seguido de febre (18,1\%), trauma (8,3\%) e de patologia dermatológica (7,8\%) (Tabela 2).

Os ECD mais requisitados no SU foram os exames de imagem (15,3\%), seguidos de ECG (12,6\%) e de estudo analítico (8,8\%). A tira-teste urinária foi realizada em 6,3\% dos episódios e o TDAR em 2,8\%. É de salientar que 47,7\% não efetuaram qualquer exame complementar de diagnóstico. Não houve necessidade de terapêutica em 68,8\% dos utentes, tendo sido executada medicação através de aerossolterapia em 6,3\%, ondansetrom oral em 4,8\% e enema em 4,5\%. A nível hospitalar, 76,9\% das crianças foram observadas por pediatria.

Relativamente às outras especialidades, as mais solicitadas foram a cirurgia geral (11,1\%), a otorrinolaringologia (4,8\%) e a ortopedia (3,8\%).

Os principais diagnósticos de saída estão representados na Tabela 3. Predominaram os diagnósticos relacionados com patologia respiratória em 29,4\% dos casos, seguido de patologia do foro gastrointestinal (25,9\%), trauma $(8,8 \%)$ e do foro de otorrinolaringologia (7,5\%). A grande maioria (92,2\%) dos utentes teve como destino a alta clínica, 6,0\% foram internados e 1,8\% transferidos para outro hospital.
TABELA 1. Características da amostra comparando entre referenciação adequada e inadequada por parte dos CSP.

\begin{tabular}{|c|c|c|c|c|}
\hline & $\begin{array}{c}\text { Total } \\
(n=398)\end{array}$ & $\begin{array}{l}\text { Referen- } \\
\text { ciação } \\
\text { adequada } \\
(\mathrm{n}=260)\end{array}$ & $\begin{array}{l}\text { Referen- } \\
\text { ciação } \\
\text { inade- } \\
\text { quada } \\
(n=138)\end{array}$ & $p$ \\
\hline \multicolumn{5}{|l|}{ Género n(\%) } \\
\hline Masculino & $198(49,7)$ & $127(48,8)$ & $73(52,9)$ & \multirow{2}{*}{$0,442^{*}$} \\
\hline Feminino & $200(50,3)$ & $133(51,2)$ & $65(47,1)$ & \\
\hline \multicolumn{5}{|l|}{ Idade (anos) n(\%) } \\
\hline Г०-1Г & $34(8,5)$ & $20(7,7)$ & $14(10,1)$ & \multirow{6}{*}{$0,001^{*}$} \\
\hline 「1-3Г & $96(24,1)$ & $48(18,5)$ & $48(34,8)$ & \\
\hline 「3-5Г & $41(10,3)$ & $24(9,2)$ & $17(12,3)$ & \\
\hline Г5-10Г & $88(22,1)$ & $56(21,5)$ & $32(23,2)$ & \\
\hline Г10-13Г & $53(13,3)$ & $40(15,4)$ & $13(9,4)$ & \\
\hline 「13-18Г & $86(21,6)$ & $72(27,7)$ & $14(10,1)$ & \\
\hline \multicolumn{5}{|l|}{ Proveniência n(\%) } \\
\hline USF/UCSP & $329(82,7)$ & $216(83,1)$ & $113(81,9)$ & \multirow{4}{*}{$0,482^{*}$} \\
\hline Fora do AceS & $51(12,8)$ & $30(11,5)$ & $21(15,2)$ & \\
\hline SAP & $11(2,8)$ & $9(3,5)$ & $2(1,4)$ & \\
\hline Ausência de dados & $7(1,8)$ & $5(1,9)$ & $2(1,4)$ & \\
\hline \multicolumn{5}{|c|}{ Triagem Manchester n(\%) } \\
\hline Não urgente & $3(0,8)$ & $3(1,2)$ & $\mathrm{O}(0,0)$ & \multirow{4}{*}{$0,420^{*}$} \\
\hline Pouco urgente & $115(28,9)$ & $68(26,2)$ & $47(34,1)$ & \\
\hline Urgente & $259(65,1)$ & $174(66,9)$ & $85(61,6)$ & \\
\hline Muito urgente & $21(5,3)$ & $15(5,8)$ & $6(4,3)$ & \\
\hline \multicolumn{5}{|c|}{ Motivo de referenciação n(\%) } \\
\hline $\begin{array}{l}\text { Pat. gastrointes- } \\
\text { tinal }\end{array}$ & $103(25,9)$ & $79(30,4)$ & $24(17,4)$ & \multirow{12}{*}{$<0,001^{*}$} \\
\hline Pat. respiratória & $77(19,3)$ & $51(19,6)$ & $26(18,8)$ & \\
\hline Febre & $72(18,1)$ & $38(14,6)$ & $34(24,6)$ & \\
\hline Trauma & $33(8,3)$ & $28(10,8)$ & $5(3,6)$ & \\
\hline Pat. dermatológica & $31(7,8)$ & $15(5,8)$ & $16(11,6)$ & \\
\hline $\begin{array}{l}\text { Pat. otorrinolarin- } \\
\text { gológica }\end{array}$ & $27(6,8)$ & $22(8,5)$ & $5(3,6)$ & \\
\hline Pat. genitourinária & $22(5,5)$ & $6(2,3)$ & $16(11,6)$ & \\
\hline Outros & $17(4,3)$ & $10(3,8)$ & $7(5,1)$ & \\
\hline Pat. oftalmológica & $13(3,3)$ & $10(3,8)$ & $3(2,2)$ & \\
\hline Pat. neurológica & $3(0,8)$ & $1(0,4)$ & $2(1,4)$ & \\
\hline Pat. infecciológica & $\mathrm{O}(0,0)$ & $\mathrm{O}(0,0)$ & $\mathrm{O}(0,0)$ & \\
\hline Pat. mental & $\mathrm{O}(0,0)$ & $\mathrm{O}(0,0)$ & $\mathrm{O}(0,0)$ & \\
\hline \multicolumn{5}{|l|}{ ECD n(\%) } \\
\hline Nenhum & $190(47,7)$ & $99(38,1)$ & $91(65,9)$ & \multirow{9}{*}{$<0,001^{*}$} \\
\hline Exames de imagem & $61(15,3)$ & $61(23,5)$ & $\mathrm{O}(0,0)$ & \\
\hline ECG & $50(12,6)$ & $50(19,2)$ & $\mathrm{O}(0,0)$ & \\
\hline Estudo analítico & $35(8,8)$ & $35(13,5)$ & $\mathrm{O}(0,0)$ & \\
\hline TTU & $25(6,3)$ & $9(3,5)$ & $16(11,6)$ & \\
\hline $\begin{array}{l}\text { TTU+ Microbio- } \\
\text { lógico }\end{array}$ & $18(4,5)$ & $1(0,4)$ & $17(12,3)$ & \\
\hline TDAR & $11(2,8)$ & $1(0,4)$ & $10(7,2)$ & \\
\hline Microbiológico & $5(1,3)$ & $1(0,4)$ & $4(2,9)$ & \\
\hline > 1 exame & $3(0,8)$ & $3(1,2)$ & $\mathrm{O}(0,0)$ & \\
\hline
\end{tabular}


TABELA 1. Características da amostra comparando entre referenciação adequada e inadequada por parte dos CSP. (cont)

\begin{tabular}{|c|c|c|c|c|}
\hline & $\begin{array}{c}\text { Total } \\
(\mathrm{n}=398)\end{array}$ & $\begin{array}{l}\text { Referen- } \\
\text { ciação } \\
\text { adequada } \\
(\mathrm{n}=260)\end{array}$ & $\begin{array}{l}\text { Referen- } \\
\text { ciação } \\
\text { inade- } \\
\text { quada } \\
(\mathrm{n}=138)\end{array}$ & $p$ \\
\hline \multicolumn{5}{|l|}{ Terapêutica n(\%) } \\
\hline Nenhum & $274(68,8)$ & $150(57,7)$ & $124(89,9)$ & \multirow{13}{*}{$<0,001^{*}$} \\
\hline Aerossolterapia & $25(6,3)$ & $25(9,6)$ & $\mathrm{O}(0,0)$ & \\
\hline Paracetamol oral & $21(5,3)$ & $21(8,1)$ & $\mathrm{O}(0,0)$ & \\
\hline Ondansetrom & $19(4,8)$ & $19(7,3)$ & $\mathrm{O}(0,0)$ & \\
\hline Enema & $18(4,5)$ & $18(6,9)$ & $\mathrm{O}(0,0)$ & \\
\hline $\begin{array}{l}\text { Outra } \\
\text { medicação oral }\end{array}$ & $9(2,3)$ & $4(1,5)$ & $5(3,6)$ & \\
\hline Outros & $8(2,0)$ & $8(3,1)$ & $\mathrm{O}(0,0)$ & \\
\hline > 1 atitude & $7(1,8)$ & $4(1,5)$ & $3(2,2)$ & \\
\hline $\begin{array}{l}\text { Aspiração secre- } \\
\text { ções }\end{array}$ & $6(1,5)$ & $6(2,3)$ & $\mathrm{O}(0,0)$ & \\
\hline Medicação IM & $5(1,3)$ & $\mathrm{O}(0,0)$ & $5(3,6)$ & \\
\hline Medicação EV & $4(1,0)$ & $4(1,5)$ & $O(0,0)$ & \\
\hline Corticoide inalado & $1(0,3)$ & $1(0,4)$ & $\mathrm{O}(0,0)$ & \\
\hline Corticoide oral & $1(0,3)$ & $\mathrm{O}(0,0)$ & $1(0,7)$ & \\
\hline \multicolumn{5}{|c|}{ Diagnóstico de saída n(\%) } \\
\hline Pat. respiratória & $117(29,4)$ & $75(28,8)$ & $42(30,4)$ & \multirow{12}{*}{$<0,001^{*}$} \\
\hline $\begin{array}{l}\text { Pat. gastrointes- } \\
\text { tinal }\end{array}$ & $103(25,9)$ & $79(30,4)$ & $24(17,4)$ & \\
\hline Trauma & $35(8,8)$ & $31(11,9)$ & $4(2,9)$ & \\
\hline $\begin{array}{l}\text { Pat. otorrinolarin- } \\
\text { gológica }\end{array}$ & $30(7,5)$ & $21(8,1)$ & $9(6,5)$ & \\
\hline Febre & $25(6,3)$ & $15(5,8)$ & $10(7,2)$ & \\
\hline Outros & $24(6,0)$ & $10(3,8)$ & $14(10,1)$ & \\
\hline Pat. genitourinária & $18(4,5)$ & $4(1,5)$ & $14(10,1)$ & \\
\hline Pat. dermatológica & $17(4,3)$ & $8(3,1)$ & $9(6,5)$ & \\
\hline Pat. infecciológica & $13(3,3)$ & $4(1,5)$ & $9(6,5)$ & \\
\hline Pat. oftalmológica & $10(2,5)$ & $9(3,5)$ & $1(0,7)$ & \\
\hline Pat. neurológica & $4(1,0)$ & $2(0,8)$ & $2(1,4)$ & \\
\hline Pat. mental & $2(0,5)$ & $2(0,8)$ & $\mathrm{O}(0,0)$ & \\
\hline \multicolumn{5}{|l|}{ Especialidade n(\%) } \\
\hline Pediatria & $306(76,9)$ & $168(64,6)$ & 138(100) & \multirow{6}{*}{$<0,001^{*}$} \\
\hline Cirurgia & $44(11,1)$ & $44(16,9)$ & $\mathrm{O}(0,0)$ & \\
\hline ORL & $19(4,8)$ & $19(7,3)$ & $\mathrm{O}(0,0)$ & \\
\hline Ortopedia & $15(3,8)$ & $15(5,8)$ & $\mathrm{O}(0,0)$ & \\
\hline Oftalmologia & $8(2,0)$ & $8(3,1)$ & $O(0,0)$ & \\
\hline Ginecologia & $6(1,5)$ & $6(2,3)$ & $\mathrm{O}(0,0)$ & \\
\hline \multicolumn{5}{|l|}{ Destino de alta n(\%) } \\
\hline Alta clínica & $367(92,2)$ & $229(88,1)$ & 138(100) & \multirow{3}{*}{$<0,001^{*}$} \\
\hline Internamento & $24(6,0)$ & $24(9,2)$ & $\mathrm{O}(0,0)$ & \\
\hline Outro Hospital & $7(1,8)$ & $7(2,7)$ & $O(0,0)$ & \\
\hline
\end{tabular}

*teste de Qui-quadrado

USF/UCSP: unidades de saúde familiar/ unidades de cuidados saúde personalizados; SAP: serviços de atendimento permanente; Pat.: patologia; ECD: exames complementares de diagnóstico; ECG: eletrocardiograma; TTU: tira-teste urinária; TDAR: teste diagnóstico antigénico rápido; IM: intramuscular; EV: endovenosa. Ausência de dados: sem informação do local dos CSP de onde eram provenientes. Outra medicação oral: outrosanalgésicos/antipirético e anti-histamínicos.
TABELA 2. Motivo de referenciação mais frequente.

\begin{tabular}{|c|c|c|}
\hline Patologia & $\begin{array}{l}\text { Motivo de } \\
\text { referenciação }\end{array}$ & $\mathrm{n}(\%)$ \\
\hline \multirow{10}{*}{$\begin{array}{l}\text { Gastrointestinal } \\
\text { (GI) }\end{array}$} & & $103(100,0 \%)$ \\
\hline & Dor abdominal & $42(40,8 \%)$ \\
\hline & Sintomas Gl & $23(22,3 \%)$ \\
\hline & Vómitos & $18(17,5 \%)$ \\
\hline & Icterícia & $10(9,7 \%)$ \\
\hline & Obstipação & $4(3,9 \%)$ \\
\hline & Diarreia & $3(2,9 \%)$ \\
\hline & Hematoquézia & $1(1,0 \%)$ \\
\hline & Fezes com muco & $1(1,0 \%)$ \\
\hline & Engasgamento & $1(1,0 \%)$ \\
\hline \multirow[t]{9}{*}{ Respiratória } & & $77(100,0 \%)$ \\
\hline & Dispneia & $22(28,6 \%)$ \\
\hline & Tosse & $17(22,1 \%)$ \\
\hline & Sintomas respiratórios & $12(15,6 \%)$ \\
\hline & Odinofagia & $9(11,7 \%)$ \\
\hline & $\begin{array}{l}\text { Síndrome dificuldade } \\
\text { respiratória }\end{array}$ & $6(7,8 \%)$ \\
\hline & Dor torácica & $5(6,5 \%)$ \\
\hline & Hemoptise & $4(5,2 \%)$ \\
\hline & Bronquiolite & $2(2,6 \%)$ \\
\hline \multirow[t]{6}{*}{ Dermatológica } & & $31(100,0 \%)$ \\
\hline & Erupção cutânea & $17(54,8 \%)$ \\
\hline & $\begin{array}{l}\text { Sinais inflamatórios } \\
\text { locais }\end{array}$ & $7(22,6 \%)$ \\
\hline & Tumefação cervical & $3(9,7 \%)$ \\
\hline & Alteração unha & $2(6,5 \%)$ \\
\hline & Sintomas pele & $2(6,5 \%)$ \\
\hline
\end{tabular}

Consideraram-se 65,3\% das referenciações como adequadas sempre que foram realizados ECD não disponíveis nos CSP (exame de imagem 23,5\%, ECG 19,2\% e estudo analítico 13,5\%); instituição de terapêutica indisponível nos CSP, ou necessidade de reavaliação após o momento da consulta (como a terapêutica oral com ondansetrom (7,3\%), o enema (6,9\%), a aerossolterapia (9,6\%), a terapêutica EV (1,5\%), ou outras atitudes terapêuticas como a aspiração de secreções (2,3\%), corticoide inalado $(0,4 \%)$; observação por outra especialidade que não a pediatria $(35,4 \%)$ e a necessidade de internamento $(6,0 \%)$ ou transferência para outro hospital $(1,8 \%)$.

A análise de concordância foi realizada segundo o teste de kappa, tendo-se registado uma concordância forte entre o motivo de referenciação e o diagnóstico de saída na patologia gastrointestinal, otorrinolaringológica, genitourinária, trauma e oftalmológica (Tabela 4).

Crianças com referenciação classificada como inadequada tinham uma mediana de idades significativamente inferior à mediana de idades do grupo classificado como referenciação adequada ( 3 anos vs 8 anos, $p<0,001$ ). A 
TABELA 3. Diagnóstico de saída mais frequente.

\begin{tabular}{|c|c|c|}
\hline Patologia & Diagnóstico de saída & $n(\%)$ \\
\hline \multirow[t]{18}{*}{ Respiratória } & & $117(100,0 \%)$ \\
\hline & Nasofaringite & $27(23,1 \%)$ \\
\hline & Amigdalite & $18(15,4 \%)$ \\
\hline & Bronquiolite & $16(13,7 \%)$ \\
\hline & Asma & $10(8,5 \%)$ \\
\hline & Broncoespasmo & $9(7,7 \%)$ \\
\hline & Tosse & $8(6,8 \%)$ \\
\hline & Pneumonia & $7(6,0 \%)$ \\
\hline & Laringite & $7(6,0 \%)$ \\
\hline & Bronquite & $4(3,4 \%)$ \\
\hline & Dor torácica & $3(2,6 \%)$ \\
\hline & Faringite & $2(1,7 \%)$ \\
\hline & Adenoidite & $1(0,9 \%)$ \\
\hline & Derrame pleural & $1(0,9 \%)$ \\
\hline & Dispneia & $1(0,9 \%)$ \\
\hline & Dor garganta & $1(0,9 \%)$ \\
\hline & Gripe & $1(0,9 \%)$ \\
\hline & Sinusite & $1(0,9 \%)$ \\
\hline \multirow[t]{14}{*}{ Gastrointestinal } & & $103(100,0 \%)$ \\
\hline & Dor abdominal & 33(32,0\%) \\
\hline & Gatroenterite aguda & $23(22,3 \%)$ \\
\hline & Vómitos & $15(14,6 \%)$ \\
\hline & Apendicite & $8(7,8 \%)$ \\
\hline & Icterícia & $6(5,8 \%)$ \\
\hline & Obstipação & $6(5,8 \%)$ \\
\hline & Quisto pilonial & $3(2,9 \%)$ \\
\hline & Diarreia & $4(3,9 \%)$ \\
\hline & Abcesso anal & $1(1,0 \%)$ \\
\hline & Gengivite & $1(1,0 \%)$ \\
\hline & Linfoadenite mesentérica & $1(1,0 \%)$ \\
\hline & Refluxo gastroesofágico & $1(1,0 \%)$ \\
\hline & Sintomas GI & $1(1,0 \%)$ \\
\hline \multirow[t]{9}{*}{$\begin{array}{l}\text { Otorrinolaringo- } \\
\text { lógica }\end{array}$} & & $30(100,0 \%)$ \\
\hline & Otite média aguda & $16(53,3 \%)$ \\
\hline & Cerúmen & $6(20,0 \%)$ \\
\hline & Corpo estranho & $2(6,7 \%)$ \\
\hline & Otalgia & $2(6,7 \%)$ \\
\hline & Otite externa & $1(3,3 \%)$ \\
\hline & Epistáxis & $1(3,3 \%)$ \\
\hline & $\begin{array}{l}\text { Perda de audição } \\
\text { súbita }\end{array}$ & $1(3,3 \%)$ \\
\hline & $\begin{array}{l}\text { Transtorno ouvido } \\
\text { externo }\end{array}$ & $1(3,3 \%)$ \\
\hline
\end{tabular}

TABELA 4. Análise de concordância - Teste de Kappa.

\begin{tabular}{|l|l|}
\hline Motivo e diagnóstico de saída & kappa \\
\hline Patologia gastrointestinal & $0,777^{*}$ \\
\hline Patologia respiratória & $0,583^{*}$ \\
\hline Febre & $0,306^{*}$ \\
\hline Trauma & $0,736^{*}$ \\
\hline Patologia dermatológica & $0,559^{*}$ \\
\hline Patologia otorrinolaringológica & $0,792^{*}$ \\
\hline Patologia genitourinária & $0,684^{*}$ \\
\hline Outros & $0,338^{*}$ \\
\hline Patologia oftalmológica & $0,776^{*}$ \\
Patologia neurológica & $0,568^{*}$ \\
\hline$p<0,01$ & \\
\hline
\end{tabular}

TABELA 5. Análise multivariada para referenciação inadequada (regressão logística).

\begin{tabular}{l|c|c|c} 
Diagnóstico de saída & OR $^{*}$ & IC 95\% & $p$ \\
$\begin{array}{l}\text { Patologia } \\
\text { gastrointestinal }\end{array}$ & 0,598 & $0,35-1,02$ & $p=0,059$ \\
$\begin{array}{l}\text { Patologia respiratória } \\
\text { Febre }\end{array}$ & 0,937 & $0,59-1,5$ & $p=0,788$ \\
$\begin{array}{l}\text { Trauma } \\
\begin{array}{l}\text { Patologia } \\
\text { infecciológia }\end{array}\end{array}$ & 0,925 & $0,39-2,17$ & $p=0,857$ \\
$\begin{array}{l}\text { Patologia } \\
\text { genitourinária }\end{array}$ & 3,49 & $0,09-0,85$ & $p=0,024$ \\
*ajustado à idade (anos) & 11,5 & $3,48-38,18,85$ & $p=0,045$ \\
\end{tabular}

análise multivariada ajustada à idade é representada na Tabela 5.

\section{DISCUSSÃO}

O estudo obteve uma taxa de referenciação adequada de $65,3 \%$ que vai de encontro ao observado noutros estudos portugueses realizados com critérios similares, mas ainda assim com alguma distância dos valores por eles apresentados de 48\% e 82\%. ${ }^{4.5}$ Esta diferença pode justificar-se pela região geográfica, pelo período de estudo dos diferentes artigos e pelas alterações estruturais que têm sido realizadas nos CSP de forma a serem capazes de dar resposta a um maior número de situações. Estas alterações estruturais compreendem a reforma nos CSP, que tem vindo a ser implementada nos últimos anos, capacitando os seus profissionais na prestação de uma maior e mais adequada resposta aos seus utentes.

Os autores salientam a mediana de idades no grupo com referenciação inadequada, significativamente inferior à mediana de idades da referenciação adequada, o que pode evidenciar que esta faixa etária suscita mais 
dúvidas pela menor especificidade das queixas. Assim, mesmo ajustando à idade, as patologias infecciosa e genitourinária mantêm-se como as mais frequentes da referenciação inadequada (Tabela 5).

Em relação aos diagnósticos de saída, observámos que os mais frequentes foram a patologia do foro gastrointestinal, respiratória, otorrinolaringológica e trauma, o que está de acordo com a literatura.,2,45

Da análise dos motivos de referenciação e diagnóstico de saída salienta-se a febre que representa 18,1\% dos motivos de referenciação e apenas 6,3\% no diagnóstico de saída. Das 72 crianças referenciadas por febre, 37 foram classificadas como patologia respiratória, 5 como patologia gastrointestinal, 3 como patologia otorrinolaringológica e 2 como patologia genitourinária, sendo os restantes 7 outros diagnósticos. Esta diferença pode ser explicada pelo recurso aos ECD disponíveis no SU, obtendo assim uma maior segurança no diagnóstico. No entanto, 18 mantiveram o diagnóstico de febre, podendo este facto ser justificado pela precocidade com que os pais procuram os serviços de saúde, por vezes impossibilitando a atribuição de um diagnóstico numa fase inicial da doença febril.

No que respeita à patologia respiratória, esta representou 19,3\% dos motivos de referenciação e 29,4\% dos diagnósticos de saída. Dos utentes com motivo de referenciação por patologia respiratória, 66 mantiveram o mesmo diagnóstico de saída, 2 foram diagnosticados como patologia otorrinolaringológica, 2 como patologia infecciosa, e 1 por febre.

No sentido de diminuir o número de casos com necessidade de referenciação hospitalar, os CSP realizam vários exames complementares de diagnóstico e terapêuticas. Os CSP e os SAP dispõem de recursos para a orientação de patologias agudas comuns nesta faixa etária, como por exemplo, a colocação de sacos coletores de urina para a realização de tira-teste urinária, no caso de suspeita de infeção do trato urinário, e o TDAR na confirmação diagnóstica de uma amigdalite por SGA, correspondendo neste estudo, ao terceiro (12\%) e quarto $(5,3 \%)$ exames mais realizados no SU, respetivamente. Estes ECD estão disponíveis nos CSP, são realizados num curto espaço de tempo e podem ser executados com a ajuda da equipa de enfermagem. Apenas uma USF do AceS de Famalicão dispõe de um eletrocardiógrafo e nenhuma dispõe de análises sanguíneas ou exames de imagem. No que concerne ao tratamento em situações agudas, os CSP têm terapêuticas orais disponíveis como antipiréticos, anti-histamínicos, ondansetrom, penicilina IM, aerossolterapia e enemas. No entanto, dada a orga- nização dos CSP onde todas as consultas são agendadas por patamares em horário fixo, incluindo a consulta aberta, os médicos especialistas em Medicina Geral e Familiar deparam-se com a dificuldade de reavaliação de um utente. Desta forma, pode ser difícil a reavaliação necessária após a administração de um enema, aerossoleterapia, ondansetrom ou hidratação oral fracionada, tendo sido por isso considerado como critério adequado para referenciação ao SU. Uma reorganização nos CSP, através da criação de um patamar de reavaliação da consulta aberta poderia diminuir o número de utentes referenciados aos cuidados hospitalares.

Uma das limitações neste estudo foi a classificação do motivo de referenciação, uma vez que, sendo um estudo retrospetivo não foi possível analisar todas as cartas de referenciação, traduzindo este aspeto um viés de informação. Para a realização de estudos futuros seria importante uma codificação, segundo ICD-10, dos motivos de referenciação no momento da entrada no SU. Com este estudo levantou-se também uma outra questão da necessidade de formação em codificação ou mesmo uma alteração na própria classificação ICD-10. Na colheita de dados do diagnóstico final foi recolhido o que os colegas do CHMA codificaram no diagnóstico de saída de acordo com ICD-10, e os autores objetivaram diagnósticos como broncospasmo, bronquiolite e asma, podendo haver aqui o viés de informação.

São também limitações o facto deste estudo ter sido realizado apenas na região de Famalicão, não permitindo extrapolar os resultados para a realidade nacional.

Tendo por base este estudo, sugere-se a implementação de algumas ferramentas que podem ser utilizadas para melhorar a comunicação entre os CSP e os cuidados hospitalares. Sugere-se a criação de uma linha telefónica para o esclarecimento de dúvidas e a criação de protocolos de referenciação nos principais motivos de referenciação de forma inadequada. Poderá também ser útil a realização de reuniões entre o serviço de pediatria e os CSP de forma a criar formação e espaço para esclarecer dúvidas nos principais motivos de referenciação.

\section{CONCLUSÃO}

A taxa de referenciação adequada aos serviços hospitalares no presente estudo foi semelhante à descrita na literatura, no entanto os autores esperavam resultados mais promissores uma vez que os estudos que existem a nível nacional foram realizados já há vários anos (20062016). Os autores salientam a concordância forte entre o motivo de referenciação e o diagnóstico de saída nas patologias gastrointestinal, otorrinolaringológica, 
trauma e oftalmológica. Com este estudo levantou-se a questão se uma reformulação nos CSP, através da criação de um patamar de reavaliação de utentes em consulta aberta, também poderia diminuir o total das referenciações para os cuidados hospitalares. No entanto, a otimização da comunicação entre os CSP e o SU, a implementação de protocolos, assim como a formação dos profissionais dos CSP, permitirão melhorar a orientação e referenciação de todas as patologias. Os autores consideram que o impacto, na melhoria da referenciação, pode não ser significativo no total de episódios no serviço de urgência pediátrico, mas otimizará a utilização de recursos de saúde pelos doentes.

\section{RESPONSABILIDADES ÉTICAS}

CONFLITOS DE INTERESSE: Os autores declaram a inexistência de conflitos de interesse na realização do presente trabalho.

FONTES DE FINANCIAMENTO: Não existiram fontes externas de financiamento para a realização deste artigo.

CONFIDENCIALIDADE DOS DADOS: Os autores declaram ter seguido os protocolos da sua instituição acerca da publicação dos dados de doentes.

PROTEÇÃO DE PESSOAS E ANIMAIS: Os autores declaram que os procedimentos seguidos estavam de acordo com os regulamentos estabelecidos pelos responsáveis da Comissão de Investigação Clínica e Ética e de acordo com a Declaração de Helsínquia revista em 2013 e da Associação Médica Mundial.

PROVENIÊNCIA E REVISÃO POR PARES: Não comissionado; revisão externa por pares.

\section{ETHICAL DISCLOSURES}

CONFLICTS OF INTEREST: The authors have no conflicts of interest to declare.

FINANCING SUPPORT: This work has not received any contribution, grant or scholarship

CONFIDENTIALITY OF DATA: The authors declare that they have followed the protocols of their work center on the publication of data from patients.

PROTECTION OF HUMAN AND ANIMAL SUBJECTS: The authors declare that the procedures followed were in accordance with the regulations of the relevant clinical research ethics committee and with those of the Code of Ethics of the World Medical Association (Declaration of Helsinki as revised in 2013).
PROVENANCE AND PEER REVIEW: Not commissioned; externally peer reviewed.

\section{REFERÊNCIAS}

1. Instituto Nacional de Estatística. [Online]. [cited 202003 21. Available from: http://www.ine.pt.

2. Caldeira T, Santos G, Pontes E, Dourado R, Rodrigues L. O dia-a-dia de uma Urgência Pediátrica. Acta Pediatr Port. 2006;37:1-4.

3. Sansa Pérez LI, Orús Escolá T, Juncosa Font S, Barredo Hernández M, Traveria Casanova J. Frecuentación a los servicios de urgências hospitalarios: motivaciones y caracteristicas de las urgencias pediátricas. An Esp Pediatr. 1996;44:97-105.

4. Pinheiro A. Referenciação à Urgência Pediátrica do Hospital S. Teotónio-Viseu. Rev Port Clin Geral. 2008;24:671-8.

5. Carvalho I, Ferreira G, Vilarinho A. Análise da referenciação ao Serviço de Urgência Pediátrico. Acta Pediatr Port. 2006;37:95-100.

6. Freitas AC, Moreira AR, Tomé S, Cardoso R. Motivos de recurso ao serviço de urgência pediátrica. Nascer Crescer. 2016;25:136-140.

7. Williams A, O'Rourke P, Keogh S. Making choices: why parents present to the emergency department for non-urgent. Arch Dis Child. 2009;94:817-20. doi: 10.1136/adc.2008.149823.

8. Haasz M, Ostro D, Scolnik D. Examining the appropriateness and motivations behind low-acuity pediatric emergency department visits. Pediatr Emerg Care. 2018;34:647-9. doi: 10.1097/ PEC.0000000000001598. 